\title{
Two Young Men with Leonine Facies and Generalized Itching - Two Case Reports of Sezary Syndrome
}

\author{
Akter $\mathbf{M}^{1^{*}}$, Khan $\mathbf{M A}^{2}$, Afrose $\mathbf{S}^{1}$, Ara $\mathrm{T}^{1}$, Ferdous $\mathbf{J}^{1}$ and Islam $\mathbf{M M}^{1}$ \\ ${ }^{1}$ Department of Haematology, Dhaka Medical College Hospital, Dhaka, Bangladesh \\ ${ }^{2}$ Bone Marrow Transplant and Haematology, Dhaka medical college hospital, Dhaka, Bangladesh
}

*Corresponding author: Akter M, Department of Haematology, Dhaka Medical College Hospital, Dhaka, Bangladesh, Tel: 88 01912112724; E-mail: mafruha673@gmail.com

Received date: Oct 14, 2014; Accepted date: Nov 21, 2014; Published date: Nov 28, 2014

Copyright: (C) 2014 Akter M, et al. This is an open-access article distributed under the terms of the Creative Commons Attribution License, which permits unrestricted use, distribution, and reproduction in any medium, provided the original author and source are credited.

\section{Summary}

Two male aged 37 years and 29 years presented with generalized itching followed by thickening and reddening of whole body for 3 and 6 months respectively. On examination generalized erythroderma, lymphadenopathy and hepato-splenomegaly were found. In both cases leucocyte count was high and of which $80-90 \%$ were morphologically Sezary cells. Peripheral blood absolute CD4+ lymphocytes were very high in both the cases. Skin biopsy showed dense infiltrate of lymphocytes in the dermis with intraepidermal collection of lymphocytes. Bone marrow was infiltrated with Sezary cells.

\section{Introduction}

Sezary syndrome (SS) is the leukaemic phase of a primary cutaneous T-cell lymphoma, Mycosis Fungoides. It is an aggressive and rare disease, 5 years disease specific survival is $<25 \%$. In peripheral blood $>1000$ Sezary cells is highly suggestive of SS. Sezary cell is a unique type of lymphocyte first described in 1938 by Sezary and Bouvrain. Sezary syndrome is triad of erythroderma, lymphadenopathy and leukaemia. SS can be diagnosed by clinical history, physical examination, $\mathrm{CBC}$ and $\mathrm{PBF}$ and skin biopsy .Incidence of primary cutaneous lymphoma increases with advancing age, the median age is $60-70 \mathrm{yrs}$ and it is rare $<30 \mathrm{yrs}$ [1]. There are three phases of developing MF/SS (a) the premycotic stage, which can be localized or diffuse with superficial eczematous or erythematous lesions; (b) the infiltrative plaque stage; and (c) the tumor stage [2]. There is another variant called mycosis d'emblée variant, in which tumors develop rapidly without a preceding premycotic or plaque stage. There is proliferation of abnormal atypical helper $\mathrm{T}$ cell In Seźary syndrome. These cell express cutaneous lymphocyte associated antigen (CLA) which helps them to adhere to cutaneous surfaces. Morphologically they are dysplastic cerebriform T cells with enlarged hyperchromatic nuclei and complex nuclear folding. Extracutaneous dissemination particularly visceral disease, is strongly associated with advanced-stage skin disease (tumors and erythroderma) and Sezary syndrome [3]. Among the extracutaneous involvement lymph node infiltration is most common. The bone marrow in MF and SS is generally thought to be spared until late in the course of the disease.

\section{Case Report 1}

A 37 years muslim male was transferred from dermatology to haematology department of $\mathrm{DMCH}$ with the complaints of intense itching and reddening and thickening of whole body surface for 3 months. There was no relieving factor and no relation with taking any food. There was no intervening period of resolution. He had no history of fever, night sweats, weight loss, jaundice, cough, joint pain, previous allergy or any other systemic symptoms. His appetite, bowel and bladder habit was normal. On examination there was generalized erythroderma and generalized lymphadenopathy. The hair distribution was normal and there was no nail abnormality. Skin was thick, nontender but there was no purpura or other lesion. There was no organomegaly. $\mathrm{CBC}$ showed $\mathrm{Hb}$ was $13.6 \mathrm{~g} / \mathrm{dL}$, WBC count was $51.35 \times 10^{9} / \mathrm{L}$, of them sezary cells with large ceribriform nuclei were $82 \%$, Total count of platelet was $388 \times 10^{9} / \mathrm{L}$ and ESR was $30 \mathrm{~mm}$ in $1 \mathrm{st}$ hr. PBF comment was suggestive of Sezary syndrome. Skin biopsy showed dense infiltrate of lymphocytes in the dermis with intraepidermal collection of lymphocytes. We have done the immunophenotyping of peripheral blood which showed CD4: CD8 ratio was 60.67 (normal ratio is $<4$ ). His bone marrow aspiration was performed and marrow was infiltrated with $40 \%$ of large lymphocytes with complex ceribriform nuclei and irregular nuclear folding. His liver function, renal function tests and USG of whole abdomen, chest X-ray were normal (Figure 1).

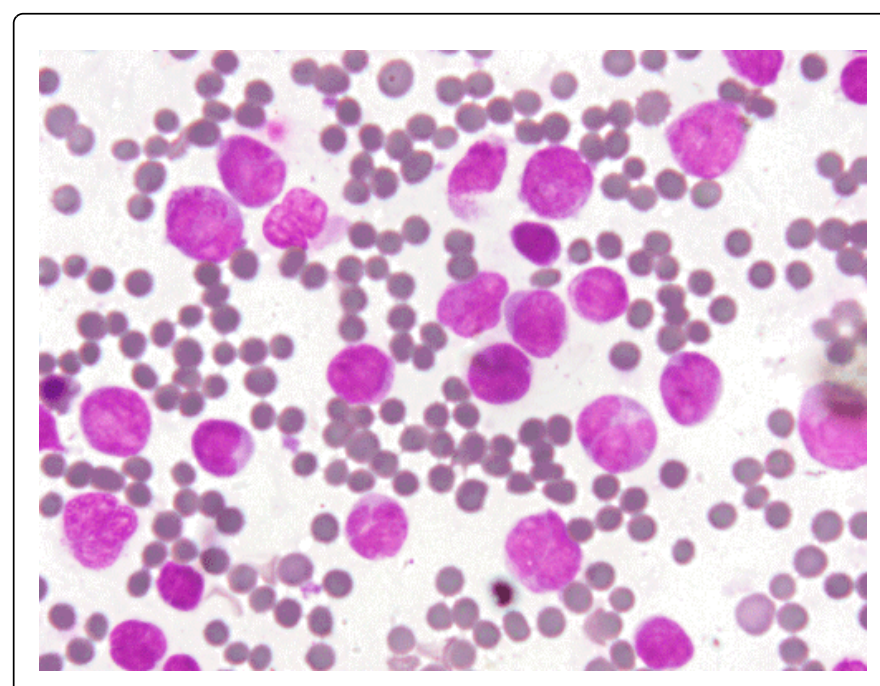

Figure 1 : Slide sezary syndrome 1

\section{Case Report 2}

A 29 years Muslim male was admitted to haematology department of DMCH with the complaints of intense itching and reddening and thickening of whole body surface for 6 months. There was no relieving factor and no relation with taking any food. He had no history of fever, night sweats, weight loss, jaundice, cough , joint pain, previous allergy 
or any other systemic symptoms. On examination there was generalized erythroderma with lionine facies, generalized lymphadenopathy and hepatosplenomegaly. The hair distribution was normal and there was no nail abnormality. Skin was thick, nontender but there was no purpura or other lesion. CBC showed Hb was 11.9 $\mathrm{g} / \mathrm{dL}, \mathrm{WBC}$ count was $65.68 \times 10^{9} / \mathrm{L}$, of them sezary cells with large ceribriform nuclei were $90 \%$, Total count of platelet was $365 \times 10^{9} / \mathrm{L}$. PBF comment was suggestive of Sezary syndrome. Skin biopsy showed dense infiltrate of lymphocytes in the dermis with intraepidermal collection of lymphocytes. Peripheral blood absolute CD4+ lymphocytes was $57349 / \mu \mathrm{L}$, (normal range is $410-1590 / \mu \mathrm{L}$ ) and ratio of CD4/CD8 was 149 , whereas normal ratio is $<4$. His bone marrow was infiltrated with $80 \%$ of large lymphocytes with complex ceribriform nuclei and irregular nuclear folding. His liver function, renal function tests and USG of whole abdomen, chest X-ray were normal (Figure 2).

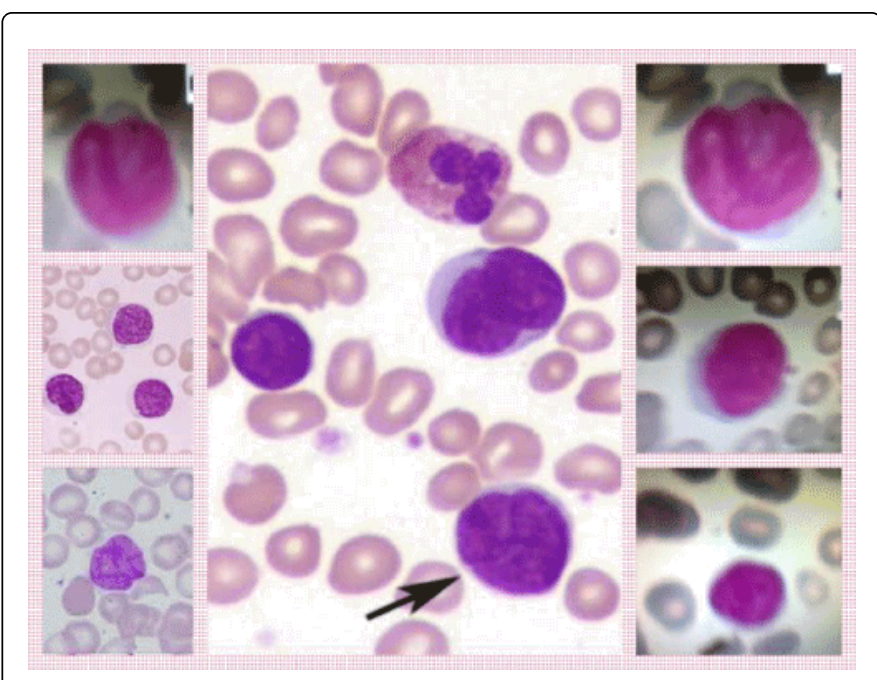

Figure 2: Slide sezary syndrome 2

\section{Discussion}

Cutaneous lymphomas are a heterogeneous group of non-Hodgkin lymphomas (NHLs) in which the skin is the primary site of involvement. They represent an entity distinct from nodal lymphomas with secondary cutaneous involvement. Primary cutaneous lymphomas usually present without signs of extracutaneous malignancy at onset of symptoms. WHO-EORTC classified cutaneous lymphomas into two broad headings: 1 . cutaneous T-cell lymphoma (CTCL) and 2. Cutaneous B cell lymphoma. Among the CTCL mycosis fungoides (MF) is most common and Sezary syndrome is the leukaemic variant of CTCL. The etiology of MF/SS remains unknown, but genetic, environmental, and infectious agents have been implicated as possible factors [4]. Human retroviruses have been suggested as possible etiologic agents in CTCL.

Mycosis fungoides usually evolves over a long period, so patients often have a long premycotic or premalignant phase with eczematous skin eruptions. The differential diagnosis during this period includes chronic eczematous, atopic or contact dermatitis, which may evolve slowly into eruptions clinically suggestive of parapsoriasis en plaque, poikiloderma atrophicans vasculare, or other benign papulosquamous skin diseases [5]. Our reported both cases were presented directly to the tumor phase of the disease. Failure of the lesions to respond to standard topical therapy may be an early clue of a different diagnosis. The earliest diagnostic phase of MF is the patch phase, characterized by scaly macules and patches that vary in size, shape, and color, tend to involve sun-protected sites, and are occasionally associated with pruritus . The tumor phase is heralded by the onset of dome-shaped, deep red to violaceous nodules emerging in areas of uninvolved skin or in pre-existing plaques [5]. The tumors may ulcerate and become secondarily infected and there is a predilection for the body folds and face, where dermal thickening, coalescing plaques, and tumors may result in characteristic "leonine facies". Generalized erythroderma may develop as the initial presenting sign of MF/SS as in our patient or may accompany plaques and tumors [6]. In Sézary syndrome (SS), the leukemic variant of CTCL, erythroderma and circulating tumor cells (Sezary cells) in the peripheral blood may be accompanied by generalized lymphadenopathy, splenomegaly, keratoderma, vitiligolike hypopigmented patches, alopecia, ectropion, nail dystrophy, and ankle edema. Intense pruritus and cutaneous pain are common in SS, our patient had intense itching but no cutaneous pain.

$\mathrm{CBC}$ and $\mathrm{PBF}$ is the first investigation which may be normal in $\mathrm{MF}$ but atypical large lymphocytes with ceribriform nucleus may be found. If these cells are $>5 \%$ of total WBC count or absolute count of these cell is $>1000 / \mathrm{mm}^{3}$ then it is suggestive of sezary syndrome, the leukemic variant of CTCL. In both the patients the total count of WBC were $>50,000 / \mathrm{mm}^{3}$ and characteristic Sezary cells were more than $80-90 \%$, that is absolute count of Sezary cells were $>1000 / \mathrm{mm}^{3}$.

Next approach is the skin biopsy which is confirmatory. Biopsy should be taken from oldest and thickest lesion, 6-mm punch biopsies are recommended. It has been recommended that steroids be discontinued for 2 to 3 weeks prior to biopsy. Punch biopsies can be divided into halves, one half for routine histology and the other half for immunophenotyping and/or molecular diagnostic studies. The characteristic atypical lymphocytes in MF and SS are dysplastic cerebriform $\mathrm{T}$ cells with enlarged hyperchromatic nuclei and complex nuclear folding. The essential criteria for diagnosis are (a) a bandlike lymphocytic infiltrate in the superficial papillary dermis, (b) epidermotropism, and (c) atypical cerebriform $\mathrm{T}$ cells in the dermal and epidermal infiltrates [7]. Pautrier microabscesses are characteristic of MF but are often absent in patch-stage lesions, erythroderma, and nonepidermotropic tumors. It is important to understand that a definitive diagnosis of MF may not be possible in some early-patchstage lesions. Multiple biopsies of separate skin lesions, immunophenotyping, and T-cell-receptor gene rearrangement studies may help confirm the diagnosis in difficult cases. Generalized exfoliative erythroderma is characteristic of Sezary syndrome but may also occur in MF. Cutaneous biopsies of erythroderma in MF/SS often lack prominent epidermo- tropism. In our skin biopsy specimens there were dense infiltrate of lymphocytes in the dermis with intraepidermal collection of lymphocytes, which was very consistent with cutaneous lymphoma.

Immunophenotyping of peripheral blood is an important investigation for staging as well as for prognosis. The immunophenotyping of the peripheral blood which showed absolute CD4+ lymphocytes was $57349 / \mu \mathrm{L}$, (normal range is $410-1590 / \mu \mathrm{L}$ ) in case 2. It indicates that there isabnormal proliferation of CD4+ helper T-cell. This is one of the criteria for cutaneous lymphoma to be considered as stage IV disease. Immunophenotyping also showed altered CD4: CD8 ratio, it was 60.67 in case 1 and 149 in case 2, whereas normal ratio is $<4$. 
Citation: Akter M, Khan MA, Afrose S, Ara T, Ferdous J, et al. (2014) Two Young Men with Leonine Facies and Generalized Itching - Two Case Reports of Sezary Syndrome. J Integr Oncol 3: 123. doi:10.4172/2329-6771.1000123

Page 3 of 3

Other investigations for staging and prognosis were also done eg. Lymph node biopsy, bone marrow aspiration and trephine biopsy, USG of abdomen, CTscan of abdomen and chest, serum LDH, $\beta-2$ microglobulin etc. S LDH were raised in both cases. Both the patients were in stage IV disease.

Prognosis of CTCL depends upon the extent of skin lesion and stage of disease. Mycosis fungoides behaves in a manner similar to other low-grade or indolent non-Hodgkin lymphomas. But patients with Sézary syndrome have a relatively poor prognosis, with a median survival of $\sim 3$ to 4 years [8]. Infection remains the most common cause of death in patients with CTCL, with Staphylococcus aureus and Pseudomonas aeruginosa being the most common pathogens infecting the skin, leading to bacteremia and sepsis [9].

Treatment options are starting from topical chemotherapy, phototherapy, systemic chemotherapy, electron beam radiotherapy, photon bean irradiation, retinoids, interferons, immunotherapy and upto stem cell transplantation.

We treated our first patient who was stage IV, with systemic chemotherapy of 6 cycles CHOP(inj. Cyclophosfamide, inj. Doxorubicin, inj. Vincristin and oral prednisolone) 21 days apart. After receiving 2 cycles of $\mathrm{CHOP}$ his generalized lymphadenopathy were regressed and intense itching was improved although erythroderma did not completely disappear at that time. After completion of CHOP therapy he was treated with PUVA (Psoralin ultraviolate A) therapy. Then he was on chlorambucil. He was apparently well for 2 years. Then he was relapsed with generalized itching, scaling of skin, nail dystrophy and maggots in the ear. Then he died with multi organ dysfunction.

We have treated the $2^{\text {nd }}$ patient with inj Fludarabine and inj Cyclophosphamide. He has completed $6^{\text {th }}$ cycles without any significant complications. He also received PUVA twice weekly for 5 months. His itching, erythroderma and exfoliation have been improved a lot. His facial configuration became near normal which was lionine facies before treatment. He is still alive with apparent good health.

\section{References}

1. Criscione VD, Weinstock MA (2007) Incidence of cutaneous T-cell lymphoma in the United States, 1973-2002. Arch Dermatol 143: 854-859.

2. Bazin E (1870) Lessons on the treatment of chronic maldies in general skin conditions en particular by the use of mineral waters compares hydro-therapy and pharmaceutical means. Paris: Adrien Delahaye.

3. Salhany KE, Greer JP, Cousar JB, Collins RD (1989) Marrow involvement in cutaneous T-cell lymphoma. A clinicopathologic study of 60 cases. Am J Clin Pathol 92: 747-754.

4. Wood GS (1995) Lymphocyte activation in cutaneous T-cell lymphoma. J Invest Dermatol 105: 105S-109S.

5. Koh HK, Charif M, Weinstock MA (1995) Epidemiology and clinical manifestations of cutaneous T-cell lymphoma. Hematol Oncol Clin North Am 9: 943-960.

6. Diamandidou E, Cohen PR, Kurzrock R (1996) Mycosis fungoides and Sezary syndrome. Blood 88: 2385-2409.

7. Smoller BR, Bishop K, Glusac E, Kim YH, Hendrickson M (1995) Reassessment of histologic parameters in the diagnosis of mycosis fungoides. Am J Surg Pathol 19: 1423-1430.

8. Marti RM, Pujol RM, Servitje O, Palou J, Romagosa V, et al. (2003) Sézary syndrome and related variants of classic cutaneous T-cell lymphoma. A descriptive and prognostic clinicopathologic study of 29 cases. Leuk Lymphoma 44: 59-69.

9. Axelrod PI, Lorber B, Vonderheid EC (1992) Infections complicating mycosis fungoides and Sézary syndrome. JAMA 267: 1354-1358. 